\title{
Acute limb ischemia in COVID-19 patients: an overview
}

\author{
Hashem Bark Abood ${ }^{1 *}$, Wael Hamed Alsaedi², Abdulwahab Mohammad Bokhari ${ }^{3}$, \\ Ahmad Mohammed Almughalleq ${ }^{4}$, Ranin Owaidh Algethami ${ }^{5}$, Sama Mohammed Zaini ${ }^{6}$, \\ Hanin Abdulrahman Ayoub ${ }^{7}$, Maan Khaled Baamer ${ }^{3}$, Wael Hassan Alanazi ${ }^{8}$, \\ Ahmed Ebraheem Al Mohammedali ${ }^{8}$, Zoha Saleh Alghamdi ${ }^{9}$
}

\author{
${ }^{1}$ Deprtment of General Surgery, Consultant of General Surgery, King Fahad Hospital-Al-Baha, KSA \\ ${ }^{2}$ College of Medicine, Umm Al Qura University-Makkah, KSA \\ ${ }^{3}$ Department of Emergency Medicine, King Faisal Hospital-Makkah, KSA \\ ${ }^{4}$ College of Medicine, Medical University of Lodz-Poland, Poland \\ ${ }^{5}$ Department of General Surgery, Hera General Hospital-Makkah, KSA \\ ${ }^{6}$ Department of General Surgery, King Fahad General Hospital-Jeddah, KSA \\ ${ }^{7}$ Department of Pathology, King Faisal Specialist Hospital and Research Center-Jeddah, KSA \\ ${ }^{8}$ College of Medicine, Vision Colleges-Riyadh, KSA \\ ${ }^{9}$ College of Medicine, King Khalid University-Abha, KSA
}

Received: 20 April 2021

Accepted: 06 May 2021

\section{*Correspondence:}

Dr. Hashem Bark Abood,

E-mail: Zina.alzahidy@ hotmail.com

Copyright: () the author(s), publisher and licensee Medip Academy. This is an open-access article distributed under the terms of the Creative Commons Attribution Non-Commercial License, which permits unrestricted non-commercial use, distribution, and reproduction in any medium, provided the original work is properly cited.

\section{ABSTRACT}

COVID-19 causes a wide range of clinical symptoms. COVID-19 hematologic and cardiovascular involvement induces thromboembolic symptoms in various organs, most notably venous thromboembolism (VTE); peripheral arterial involvement is uncommon. Acute limb ischemia (ALI) is a vascular emergency known as a dramatic drop in arterial perfusion of a limb that poses a challenge to the limb's viability. Given routine need for thromboprophylaxis, hospitalised COVID-19 patients are at an elevated risk of ALI, limb loss, or even mortality. Understanding the various specific symptoms of COVID-19 patients, especially extrapulmonary manifests, is crucial for both better patient care and the development of adequate infection prevention methods. Although ALI is a complication of COVID-19 in hospitalized patients, it is now being understood that it can arise in patients with mild COVID-19 symptoms, and that ALI can be the first presenting sign of COVID-19 even in the absence of respiratory symptoms. It is recommended that acutely admitted COVID-19 cases should be given pharmacologic thromboprophylaxis with low molecular weight heparin (LMWH) or fondaparinux unless there is a chance of bleeding or contraindicated. Anticoagulant treatment, primarily with LMWH, correlates strongly with an improved prognosis in serious COVID19 patients who follow sepsis-induced coagulopathy requirements or have significantly elevated D-dimer levels.

Keywords: COVID-19, SARS-CoV-2, Ischemia, ALI, Thrombosis

\section{INTRODUCTION}

Coronavirus disease 2019 (COVID-19) is caused by extreme acute respiratory syndrome coronavirus 2 and has been declared a global pandemic by the World health organization. ${ }^{1}$ It is a positive-sense, single-stranded, enveloped RNA virus with a helical capsid. ${ }^{2}$ Coronavirus disease 2019 (COVID-19) is a multi-systemic infection that mainly affects the respiratory, hematologic, and cardiovascular systems. ${ }^{3}$

COVID-19 causes a wide range of clinical symptoms, including bilateral pneumonia, acute respiratory distress 
syndrome (ARDS), endothelial dysfunction, hypercoagulability, and multiorgan failure. ${ }^{2}$ COVID-19 hematologic and cardiovascular involvement induces thromboembolic symptoms in various organs, most notably venous thromboembolism (VTE); peripheral arterial involvement is uncommon. ${ }^{4,5}$ Klok et al performed a study on 184 COVID-19 patients, finding that the average rate of thrombosis was 31 percent, with venous thromboembolism accounting for 27 percent of cases and arterial thrombotic incidents accounting for 3.7 percent. $^{6}$

The majority of SARS-CoV-2 patients diagnosed with limb ischemia had increased inflammatory markers and were mostly elderly. Furthermore, angiotensin-converting enzyme 2, the SARS-CoV-2 receptor, is expressed on the membrane of vascular muscle and endothelial cells, and infection of these cells can cause an inflammatory response in the blood vessel walls, predisposing to clot developing. ${ }^{7}$

Acute limb ischemia (ALI) is a vascular emergency known as a dramatic drop in arterial perfusion of a limb that poses a challenge to the limb's viability. ${ }^{8}$ The most frequent causes involve embolism from cardiac chambers, and is most often combined with atrial fibrillation or acute myocardial infarction, embolism from arterial aneurysms, thrombosis of native limb arteries or artery grafts, iatrogenic thromboembolism after vascular procedures, aortic dissection, and traumatic vascular injury. ${ }^{9}$ COVID-19 patients who present with acute lower extremity ischemia caused by arteriosclerosis obliterans and venous thrombosis at the same time have a poor prognosis and a higher mortality rate. ${ }^{10}$

There has been an increase in the prevalence of ALI in COVID-19 patients. $^{11}$ COVID-19 patients present with acute lower extremity ischemia due to arteriosclerosis obliterans and venous thrombosis at the same time have a worse prognosis and a higher mortality rate. The prevalence of ALI correlated with COVID-19 patients that need hospitalisation varies from $3 \%$ to $15 \% .^{12,13}$

\section{CURRENT BURDEN}

Evolving findings of vascular skin results in COVID-19 patients increase the risk infection could present with the sequelae of a virally induced hypercoagulable state, possibly accompanying or in the absence of concomitant respiratory symptoms. Infection can also reveal a hypercoagulable condition in patients with pre-existing risk factors such as diabetes, which leads to endothelial dysfunction and increased platelet activation. ${ }^{14}$ In Saudi Arabia, a study at King Abdullah university hospital (KAUH) involved 1300 COVID-19 patients; $0.54 \%$ of patients had acute lower limb ischemia (ALLI). (28.57\%) of had asymptomatic COVID-19 infection and (71.43\%) of them developed ALLI during their admission due to COVID-19. ${ }^{15}$
Furthermore, the study's results revealed an improvement in ALLI among COVID-19 patients in ICUs (two cases in the last year compared to five cases in two months). ${ }^{16} \mathrm{~A}$ retrospective research was undertaken in Italy to investigate the prevalence and outcomes of 20 patients with acute limb ischemia. According to the results, the rate of acute limb ischemia was higher in the first few months of 2020 compared to 2019, and the higher incidence was attributed to an increase in cases triggered by thromboembolic disease associated with COVID-19. ${ }^{17}$ Bellosta et al conducted a single-center, retrospective cohort analysis and discovered that the incidence of ALI increased dramatically during the COVID-19 pandemic. $^{17}$

Topcu et al performed a case study and found that COVID-19 infection is associated with increased occurrence of thromboembolic events, such acute limb ischemia (ALI). Also, young and sometimes stable patients will grow ALI despite prophylactic anticoagulation. ${ }^{18}$

Putko et al investigated the prevalence documenting COVID-19 affected patients with limb or digit ischemia; the results of the search support the hypothesis that SARS-CoV-2 infection is associated with an elevated risk of limb ischemia. Although the precise pathophysiology is unclear, evidence for inflammatory-mediated thrombosis is one plausible cause, which may justify the use of immunotherapy in combination with anticoagulation for the treatment and/or prevention of thromboembolic events. ${ }^{19}$

Kartikasari et al published a case report and discovered a connection between COVID -19 infections and coagulopathy, which can occur as acute limb ischemia. This study is intended to improve understanding of hypercoagulation in COVID -19 patients and acute limb ischemia as a potential manifestation. ${ }^{20}$

\section{PATHOGENESIS}

In SARS-CoV-2 patients, so many researchers noted an inflammatory-mediated thrombosis pathway. To explain this effect, various mechanisms have been suggested. Several authors have identified an inflammatory-mediated thrombosis pathway in SARS-CoV-2 patients. ${ }^{21,22}$ This pro-inflammatory condition is induced not only by inflammatory cytokines that induce a pro-thrombotic state, but it is also suspected that SARS-CoV-2 will directly infect endothelial cells through the alveolar ACE2 receptor, resulting in both endothelial cell activation and dysfunction. ${ }^{23}$ The arterial and venous thromboembolic events seen in COVID-19 patients are believed to be the result of a dysregulated hyperinflammatory reaction. COVID-19 patients have consuming coagulopathy, which is close to sepsisassociated disseminated intravascular coagulopathy (DIC). ${ }^{24-25}$ There is a rise in D-dimer, fibrinogen, coagulation factors, acquired antiphospholipid antibodies, 
and a decline in protein $\mathrm{C}$, protein $\mathrm{S}$, antithrombin, and platelet and neutrophil hyperactivation. ${ }^{26}$ It is likely that the elevated rate of arterial thromboembolic events in COVID-19 patients is caused by a composite of these pathways, rather than just one.

Furthermore, several studies show that severe COVID-19 infections increase the number of helper $\mathrm{T}$ lymphocytes, which contain an abundance of CD14 and CD16; these monocytes then promote the formation of IL-6, a central mediator in COVID-19. IL-6 is a cytokine that stimulates the development of acute-phase reactants (APRs) in the liver, such as CRP and fibrinogen. These two APRs play an important role in making the body hypercoagulable, which contributes to thrombosis. ${ }^{2}$ Putko et al found increased inflammatory factors and/or changed clotting parameters in the vast majority of patients. This was true particularly in patients who did not have any major baseline comorbidities. ${ }^{19}$

\section{DISCUSSION}

Limb ischemia in SARS-CoV-2 positive patients poses a novel risk to physicians who treat extremity injuries. In the treatment of patients with COVID-19-associated ALI, prompt vascular surgery consultation is important. ${ }^{24}$

Although ALI is a complication of COVID-19 in hospitalized patients, it is now being understood that it can arise in patients with mild COVID-19 symptoms, and that ALI can be the first presenting sign of COVID-19 even in the absence of respiratory symptoms. ${ }^{27}$ In a study of 49 patients diagnosed with COVID-19, 22 (45\%) showed symptoms of acute arterial ischemia before being diagnosed with COVID-19, while the remaining 27 $(55 \%)$ experienced ischemia during hospitalization. ${ }^{28}$

Infected patients with little to no COVID-19 symptoms can experience a prothrombotic state, and some studies have identified patients who complained mostly of acute extremity discomfort, which was mostly accompanied by moderate respiratory symptoms or other COVID-19 symptoms, although in some instances, ALI was the only clinical manifestation of COVID-19. ${ }^{28-34}$ ALI has also been recorded after recovering from a mild infection. ${ }^{35}$

Since arterial thrombosis may arise without the typical risk factors for ALI, a high index of suspicion is required to detect developing ALI and provide prompt, targeted care. The diagnosis of ALI is mostly clinical, relying on physical examination findings, such as assessment of extremity pressures, and the patient's medical history. Vascular imaging helps plan surgery by confirming the presence and degree of arterial obstruction. ${ }^{33-34}$

The most popular confirmatory imaging modalities are duplex ultrasound and CT angiography, which help assess the anatomic site and degree of the arterial occlusion. A proximal thromboembolic origin can be identified using CT angiography. ${ }^{36}$ The vascular imaging analysis used in the COVID-19 pandemic was based on the patient's stabilization for transition to the imaging lab, if necessary, as well as concern for resource distribution. Just 8 patients with acute upper or lower extremity ischemia received confirmatory imaging trials in a limited group of 16 patients. ${ }^{36}$ In addition, there is normally sufficient time to undergo vascular imaging prior to surgery for patients with viable or partially endangered limbs. Patients that are in imminent danger of losing a limb, on the other hand, need more immediate assessment, usually in an emergency room.

\section{MANAGEMENT INTERVENTIONS}

Based on the patient's general health, degree of ischemia, and limb viability, it must be determined if surgery is necessary and, if so, if an endovascular or open solution should be used. When seeking action, it is important to understand the seriousness of systemic disease. ${ }^{34}$ Open thrombectomy is the primary surgical revascularization procedure for ALI, while other adjuncts such as endarterectomy/patch angioplasty and/or surgical bypass may be available. Endovascular revascularization can involve catheter-directed thrombolysis or percutaneous mechanical thrombectomy (a variety of devices are obtainable). ${ }^{37}$ A combination of open and endovascular approaches may be required in some cases. In one case of ALI caused by severe thrombosis of an aortic aneurysm, iliac arteries, and infrainguinal arteries, thrombolytic therapy preserved aortoiliac occlusion patency and bilateral open thrombectomy of the infrainguinal vessels restored distal perfusion. ${ }^{38}$

In addition, all COVID-19 cases admitted to the hospital must be given pharmacologic thromboprophylaxis with low molecular weight heparin (LMWH) or fondaparinux unless there is a chance of bleeding and complete therapeutic-intensity anticoagulation in the right clinical situation. ${ }^{39}$ Anticoagulant treatment, primarily with LMWH, correlates strongly with an improved prognosis in serious COVID-19 patients who follow sepsis-induced coagulopathy requirements or have significantly elevated D-dimer levels. ${ }^{40}$

According to Sánchez et al thromboembolectomy was performed using an embolectomy catheter sized according to the location of occlusion. Anticoagulation therapy with low-molecular-weight heparin (LMWH) and UFH during hospitalisation and with LMWH was started for patients as a bridging treatment to warfarin at discharge. ${ }^{40}$ An analysis of 449 COVID-19 patients found that those given prophylactic doses of heparin and enoxaparin had a statistically significant reduction in deaths (40.0 percent experimental, 64.2 percent control) ${ }^{40}$

\section{CONCLUSION}

COVID-19 is an outbreaking multi-systemic disorder affecting the hematologic and cardiovascular systems. Given routine need for thromboprophylaxis, hospitalized 
COVID-19 patients are at an elevated risk of ALI, limb loss, or even mortality. Understanding the various specific symptoms of COVID-19 patients, especially extrapulmonary manifests, is crucial for both better patient care and the development of adequate infection prevention methods.

Funding: No funding sources

Conflict of interest: None declared

Ethical approval: Not required

\section{REFERENCES}

1. Gomez-Arbelaez D, Ibarra-Sanchez G, GarciaGutierrez A, Comanges-Yeboles A, AnsuateguiVicente M, Gonzalez-Fajardo JA. COVID-19-related aortic thrombosis: a report of four cases. Ann Vasc Surg. 2020;67:10-3.

2. Hasan SA, Haque A, Nazir F. Acute Limb Ischemia: A Rare Complication of COVID-19. Cureus. 2020;12(11):e11488.

3. Roberts CM, Levi M, McKee M. COVID-19: a complex multisystem disorder. $\mathrm{Br} \mathrm{J}$ Anaesth. 2020;125:238-42.

4. Cheruiyot I, Kipkorir V, Ngure B. Arterial thrombosis in Coronavirus Disease 2019 patients: a rapid systematic review. Ann Vasc Surg. 2021;70:273-81.

5. Bilaloglu S, Aphinyanaphongs $\mathrm{Y}$, Jones $\mathrm{S}$. Thrombosis in hospitalized patients with COVID-19 in a New York City health system. JAMA. 2020;324:799-801.

6. Klok F, Kruip M, van der Meer N. Incidence of thrombotic complications in critically ill ICU patients with COVID-19. Thromb Res. 2020;191:145-7.

7. Shalbub S. The mystery of COVID-19-associated arterial thrombosis. J Vasc Surg. 2020.

8. Han H., Yang L., Liu R. Prominent changes in blood coagulation of patients with SARS-CoV-2 infection. Clin Chem Lab Med. 2020;58(7):1116-20.

9. Frohman EM, Villemarette-Pittman NR, Melamed E. Part I. SARS-CoV-2 triggered 'PANIC' attack in severe COVID-19. J Neurol Sci. 2020;116936.

10. Björck M, Earnshaw JJ, Acosta S, Gonçalves FB, Frederic C, Debus ES, Hinchliffe R, Jongkind V, Koelemay MJW, Menyhei G, Svetlikov AV, Tshomba Y, Van Den Berg JC. Editor's choiceEuropean society for vascular surgery (ESVS) 2020 clinical practice guidelines on the management of acute limb ischaemia. Eur J Vasc Endovasc Surg. 2020;59(2):173-218.

11. Zhou B, She J, Wang Y, Ma X. Venous thrombosis and arteriosclerosis obliterans of lower extremities in a very severe patient with 2019 novel coronavirus disease: a case report. J Thromb Thrombolysis. 2020;50:229-32.

12. Baccellieri D, Bilman V, Apruzzi L. A case of Covid-19 patient with acute limb ischemia and heparin resistance. Ann Vasc Surg. 2020;68:88-92.
13. Zhou B, She J, Wang Y, Ma X. Venous thrombosis and arteriosclerosis obliterans of lower extremities in a very severe patient with 2019 novel coronavirus disease: a case report. J Thromb Thrombolysis. 2020;50:229-32.

14. Owen T. Acute limb ischemia as sole initial manifestation of SARS-CoV-2 infection. J Vascular Surg Cases and Innovative Techn. 2020:511-3.

15. Al-Zoubi N, Shatnawi N, Jarbo H. Acute Lower Limb Ischemia in Patients Infected with COVID-19. Int J Gen Med. 2021;14:833-39.

16. Ahmad H, Shubair SM, Kruer J, Hatoum CA. Acute lower-extremity ischemia in a patient with COVID19. Am J Case Rep. 2020;21:928471.

17. Bellosta, Raffaello B. Acute limb ischemia in patients with COVID-19 pneumonia. J vascular surg vol. 2020;1020\172,6:1864-72.

18. Canl A. Acute limb ischemia in a COVID-19 patient. Thrombosis Update vol. 2021:2:100031.

19. Putko, Robert M. SARS-CoV-2 and limb ischemia: A systematic review. J clin orthop trauma. 2020.

20. Kartikasari, Ulfah. Acute limb ischemia in a patient with Covid-19 pneumonia: a case report. J thrombosis thrombolysis. 2021;1-6.

21. Abou-Ismail MY, Diamond A, Kapoor S, Arafah Y, Nayak L. The hypercoagulable state in COVID-19: incidence, pathophysiology, and management. Thromb Res. 2020;194:101-15.

22. Björck M, Earnshaw JJ, Acosta S, Gonçalves FB, Frederic C, Debus ES et al. Van Den Berg Editor's choice-European society for vascular surgery (ESVS) 2020 clinical practice guidelines on the management of acute limb ischemia. Eur J Vasc Endovasc Surg. 2020;29(2):173-218.

23. Imdes JE, Koleilat I, Hatch AN, Choinski K, Jones $\mathrm{DB}$, Aldailami $\mathrm{H}$ et al. Early experience with arterial thromboembolic complications in patients with COVID-19. J Vasc Surg. 2020.

24. Piazza G, Morrow DA. Diagnosis, management, and pathophysiology of arterial and venous thrombosis in COVID-19. JAMA. 2020;324(24):2548-9.

25. Abou-Ismail MY, Diamond A, Kapoor S, Arafah Y, Nayak L. The hypercoagulable state in COVID-19: incidence, pathophysiology, and management. Thromb Res. 2020;194:101-15.

26. Rutherford RB, Baker JD, Ernst C, Johnston KW, Porter JM, Ahn S, Jones DN. Recommended standards for reports dealing with lower extremity ischemia: revised version. J Vasc Surg. 1997;26(3):517-38.

27. Fournier M, Faille D, Dossier A. Arterial Thrombotic Events in Adult Inpatients With COVID-19. Mayo Clin Proc. 2021;96:295.

28. Etkin Y, Conway AM, Silpe J. Acute Arterial Thromboembolism in Patients with COVID-19 in the New York City Area. Ann Vasc Surg. 2021;70:290.

29. Maurera AH, Vu JH, Rehring TF. Acute Limb Ischemia in Minimally Symptomatic SARS-CoV-2 Infection. J Vasc Interv Radiol. 2020;31:2150. 
30. Kaur P, Posimreddy S, Singh B. COVID-19 Presenting as Acute Limb Ischaemia. Eur J Case Rep Intern Med. 2020;7:001724.

31. Thompson O, Pierce D, Whang D. Acute limb ischemia as sole initial manifestation of SARS-CoV2 infection. J Vasc Surg Cases Innov Tech. 2020;6:511.

32. Singh B, Kaur P, Ajdir N. Covid-19 Presenting as Acute Limb Ischemia. Cureus. 2020;12:e9344.

33. Wengerter SP, Wengerter KR, Masoudpoor H. Acute aortoiliac and infrainguinal arterial thrombotic events in four patients diagnosed with the novel coronavirus 2019. J Vasc Surg Cases Innov Tech. 2020;6:698.

34. Katzen BT. Clinical diagnosis and prognosis of acute limb ischemia. Rev Cardiovasc Med. 2002;3(2):S2-6.

35. Veerasuri S, Kulkarni SR, Wilson WR, Paravastu SCV. Bilateral Acute Lower Limb Ischemia Secondary to COVID-19. Vasc Endovascular Surg 2021;55:196.

36. Silingardi R, Gennai S, Migliari M. Acute limb ischemia in COVID-19 patients: Could aortic floating thrombus be the source of embolic complications? J Vasc Surg. 2020;72:1152.
37. Colling ME, Kanthi Y. COVID-19-associated coagulopathy: An exploration of mechanisms. Vasc Med. 2020;25:471.

38. Paranjpe I, Fuster V, Lala A. Association of treatment dose anticoagulation with in-hospital survival among hospitalized patients with COVID19. J Am Coll Cardiol. 2020;76:122-4.

39. Tang N, Bai H, Chen X, Gong J, Li D, Sun Z. Anticoagulant treatment is associated with decreased mortality in severe coronavirus disease 2019 patients with coagulopathy. J Thromb Haemost. 2020;18:1094-9.

40. Sánchez JB, Cuipal Alcalde JD, Ramos Isidro R. Acute Limb Ischemia in a Peruvian Cohort Infected by COVID-19. Ann Vasc Surg. 2020;72:196-204.

Cite this article as: Abood $\mathrm{HB}$, Alsaedi $\mathrm{WH}$, Bokhari AM, Almughalleq AM, Algethami RO, Zaini SM, et al. Acute limb ischemia in COVID-19 patients: an overview. Int J Community Med Public Health 2021;8:3151-5. 